\title{
Educação para a Criatividade em Adolescentes: Uma Experiência com Future Problem Solving Program Internacional
}

\author{
Education for Creativity in Adolescents: An Experience with the \\ Future Problem Solving Program International
}

\author{
Ivete Azevedo ${ }^{1 *}$ \\ Maria de Fátima Morais ${ }^{2}$ \\ Fernanda Martins ${ }^{3}$ \\ ${ }^{1}$ Torrance Center ${ }^{2}$ Universidade do Minho ${ }^{3}$ Universidade do Porto
}

\begin{abstract}
Os contornos atuais reforçam a importância da educação criatividade intencional, particularmente no contexto escolar. Neste contexto, a adolescência também tem drivers específicos para este tipo de educação sistemática. Enquanto isso, a promoção das competências criativas é legitimada pela investigação. Este estudo examinou os efeitos de um programa de intervenção aplicada a (Future Problem Solving International Program) internacionalmente em adolescentes portugueses. Este programa desenvolve competências de questões criativas de resolução de problemas relacionados com o futuro. Participaram 131 alunos do ensino secundário, dos 12 aos 15 anos. Chegou a um grupo experimental $(\mathrm{N}=67)$ e um grupo controle $(\mathrm{N}=64)$ e foi avaliado tanto nos dois momentos de teste pré e pós. A implementação do programa durou sete meses no contexto formal e avaliação o TTCT- Torrance Tests of Creative Thinking. Os resultados indicam benefícios estatisticamente significativos do grupo experimental em várias habilidades criativas em um índice de criatividade global e fortalecimento da resolução criativa de problemas (inovação/adaptação) estilos. Os dados são analisados no contexto de outros estudos e as possibilidades futuras desta pesquisa são apresentados.
\end{abstract}

Palabras-Clave: Criatividade, Educação, Resolução de problemas, Adolescência.

\begin{abstract}
The actuality highlight the importance of a purposeful education to creativity, particularly in the school context. In this context, adolescence also comes up with reinforcing specificities for such systematic education. For its part, the promotion of creative skills has been legitimized by the research. This study analyzed the effects of an intervention program internationally applied (Future Problem Solving Program International) in Portuguese adolescents. That program improves the creative problem solving skills considering global issues with a future perspective. Participants were 131 students from the Junior High School, (from 12 to 15 years old). It was considered an experimental group $(\mathrm{N}=67)$ and a control one $(\mathrm{N}=64)$ and both were evaluated in the pre and the posttest moments. The implementation of the program lasted seven months in extracurricular context and the evaluation used the TTCT - Torrance Tests of Creative Thinking. The results indicate gains statistically significant in the experimental group in several creative skills, in the global index of creativity, also indicating an enhancement of creative solving problem styles (innovation/adaptation). These data are discussed in the context of other studies and it is pointed future developments for this research.
\end{abstract}

Keywords: Creativity, Education, Problem solving, Adolescence.

*Contacto: iveteazevedo@tcportugal.org

ISSN: $1696-4713$

www.rinace.net/reice/

revistas.uam.es/reice
Recibido: $\quad 11$ de julio 2016

$1^{\text {a }}$ Evaluación: 28 de octubre 2016

$2^{\text {a }}$ Evaluación: 9 de diciembre 2016

Aceptado: $\quad 15$ de diciembre 2016 


\section{Fundamentação teórica}

Na década de 50, Guilford (1950) veio incentivar a pesquisa sobre criatividade, mostrando a relevância deste construto para vários domínios, incluindo a educação. O estudo da criatividade não seria mais o mesmo, crescendo, ganhando credibilidade científica e diversificando-se (Alencar, 2015). Porém, no início do séc. XXI, não só se exclama a importância da criatividade, mas se lhe reclama um estatuto de imprescindível, dadas as aceleradas e imprevisíveis transformações que o mundo impõe, ao cidadão comum e à liderança dos contextos socio-político e científico-tecnológico: não haverá forma de lidar eficazmente com tal mundo sem competências de resolução criativa de problemas (Miller e Mumford, 2014).

É então óbvia a importância de uma educação que contemple intencionalmente o desenvolvimento da criatividade, nomeadamente no contexto escolar (Trilling e Fadel, 2012). Neste contexto, a adolescência surge como período com especificidades reforçadoras de esforços para promoção sistemática da criatividade. Assim, desde os estudos desenvolvimentais de Torrance (1976) que se verifica no início da adolescência um declínio nas competências criativas, nomeadamente por haver grande centração nas expectativas do outro, fortes alterações pubertárias e cognitivas, acontecendo depois, ao longo desta faixa etária, um aumento de tais competências, ao qual a maior capacidade de abstração ou a construção de identidade não são alheios (Guinard e Lubart, 2006). Assim, quer no início, quer no desenrolar deste período, é pertinente colmatar lacunas e potenciar crescimento, respetivamente. Aliás, a nível formal, é preconizado para o $3^{\circ}$ ciclo, no sistema educativo nacional, o desenvolvimento da criatividade (Lei de Bases do Sistema Educativo, lei $\mathrm{n}^{\circ} 46 / 86$ de 14 de Outubro).

Por seu lado, a promoção de competências criativa, apesar de não ser ainda um tópico totalmente pacífico, é atualmente legitimada, nos seus efeitos, pela investigação. Estudos de meta-análise (Ma, 2006; Scott, Leritz e Mumford, 2004) vêm mostrar resultados positivos de programas de intervenção, enfatizando a noção de um potencial criativo inerente a todos os indivíduos e passível de ser desenvolvido de forma sistemática (Runco, 2014). Neste sentido, existe diversidade de estratégias e de programas de treino face a competências de resolução criativa de problemas, entendendo esta como tradutora de ideias simultaneamente eficazes e originais (Runco e Jaeger, 2012).

Na aposta face à promoção da criatividade, um dos programas que se tem imposto durante décadas, quer em diversidade de países nos quais é aplicado, quer em estudos que vai implicando (Cramond, 2009), é o Future Problem Solving Program International FPSPI (Torrance, Torrance, Williams e Horng, 1978). Nasceu nos Estados Unidos da América, mas atualmente envolve países tão diversos como Turquia, Singapura, Inglaterra ou Austrália. Existe mesmo, todos os anos, uma competição internacional (www.fpspi.org), na qual crianças e jovens de todos os continentes apresentam um projeto criativo desenvolvido no âmbito da aplicação do programa. Este programa tem como objetivo principal o treino de competências criativas (em crianças e jovens, mas também nos adultos aplicadores do programa), através da abordagem de questões futuristas, ou seja, facilitando o exercício de competências de resolução criativa de problemas a partir de temas atuais, posicionados numa perspetiva futurista. Esta abordagem, permitindo projetar e refletir futuros alternativos, e assim facilitar a gestão da incerteza que cada vez mais tal futuro acarreta, torna-se uma ferramenta essencial 
para um pensamento estratégico, criativo e crítico, assim como reflexivo de conhecimentos (Masini, 2011).

O FPSPI baseia-se na metodologia CPS (Creative Problem Solving) desenvolvida por autores como Isaksen, Dorval e Treffinger (2011). Esta metodologia propões seis etapas de resolução de problemas, desde a definição do problema até à comunicação da solução para esta ser aceite, implicando sempre a aplicação dos pensamentos divergente e convergente, dominando cada um deles em etapas específicas. Baseia-se ainda no pressuposto de que um problema tem sempre solução: é então, e somente, um desafio ao indivíduo que pretende resolvê-lo.

O percurso do FPSPI desenrola-se também no decurso de seis etapas, embora não totalmente coincidentes com as do CPS. Inicialmente define-se um problema, ainda genérico -a identificação da área problema; operacionaliza-se depois o problema, já concreto, a resolver- a seleção do problema; seguem-se a produção e a seleção de critérios de decisão para, na fase seguinte, haver a avaliação das soluções propostas; por último, estando selecionada uma ideia-solução, avança-se para um plano de implementação da solução.

A aplicação do FPSPI pode acontecer em três modalidades competitivas (Global Issues Problem Solving, Comunity Problem Solving e Scenario Writing) e uma não competitiva (Action Base Problem Solving). A Resolução Criativa de Problemas na Comunidade (Comunity Problem Solving) implica que um grupo sem número definido de participantes identifique e crie uma proposta de solução para um problema da comunidade (local, regional, mundial). Já a Resolução Criativa de Problemas Globais Futuristas (Global Issues Problem Solving) envolve equipas com máximo de 6 elementos e o problema, contextualizado em domínios como a educação, a cultura, o ambiente, a saúde ou os serviços humanitários, é colocado através de uma História Problema Futurista, esperando-se que a resolução criativa do problema se expresse num relatório específico (Booklet/Livreto CriAtivo). Individualmente, jovens participantes podem exercitar o FPSPI através da modalidade Escrita de Cenários Futuristas (Scenario Writing), escrevendo textos criativos face a contextos que envolvem uma visão de, pelo menos, 25 anos no futuro. O FPSPI é ainda um bom método para ser usado na aprendizagem de outros conteúdos (Cramond e Fairweather, 2013), sendo este o principal objetivo da modalidade não competitiva Action Base Problem Solving (Resolução Criativa de Problemas em Ação). Esta modalidade desenvolve-se sempre em grupo (sem número limite de participantes), os problemas a resolver podem advir de histórias, textos académicos, notícias de jornal ou qualquer outro suporte informativo. Tal como nas restantes modalidades, os trabalhos submetidos a um comité de avaliadores, receberão um relatório de progressão de competências, com o qual é possível promover a aprendizagem a partir do erro. Para todas as modalidades, há um mentor, adulto e com formação no programa, que conduz a aplicação. Esta aplicação pode ocorrer em contextos educativos formais (escola, em sala de aula ou extra-curricularmente) ou não formais (família, autarquias, ...)

Num processo que dura, um ano letivo, os participantes vão treinando os pensamentos criativos, crítico e futurista, face a um problema bem definido e cuja solução será operacionalizada num plano de implementação. Paralela e obviamente, são treinadas competências de competição e de cooperação, estando em causa, por exemplo, a gestão do tempo, de emoções ou a tomada de decisão. Treinam-se ainda competências de 
comunicação no trabalho de grupo e na apresentação do plano de solução, através de relatórios, desenhos, vídeos, ou entrevistas, por exemplo (Treffinger, Selby e Crumel, 2012).

Há estudos sobre a eficácia do FPSPI em diferentes competências. O programa parece mostrar eficácia nos pensamentos criativos, crítico e analítico (Cramond e Fairweather, 2013; Kaufman, 2012), assim como na comunicação oral e escrita (Volk, 2008), motivação para a aprendizagem (Morais, Jesus, Azevedo, Araújo e Viseu, 2015) e competências de investigação (Alvino, 1993). Especificamente em Portugal, onde o tópico da criatividade não é ainda abundante em contexto educativo, sendo-o muito menos o seu treino sistemático, torna-se de particular relevância estudar os efeitos em competências criativas de um programa tão divulgado internacionalmente como o FPSPI, sendo este o objetivo da investigação a apresentar.

\section{Método}

O estudo, de natureza quasi-experimental utilizou o design de grupo de controlo não equivalente uma vez que os sujeitos não foram aleatoriamente escolhidos mas eram elementos de duas turmas de alunos pré-existentes. Com ele se pretende analisar os efeitos do Programa Future Problem Solving em competências criativas de estudantes do $3^{\circ}$ ciclo do Ensino Básico. Coloca-se como hipótese que o grupo experimental apresentará valores significativamente mais elevados em várias competências criativas Fluência, Originalidade, Elaboração, Abstração nos Títulos, Resistência ao Fechamento, Forças Criativas- face ao grupo de controlo no $2^{\circ}$ momento da avaliação. Espera-se ainda que o grupo experimental, nesse momento e face ao de controlo, demonstre resultados significativamente mais elevados nas dimensões de Adaptação e de Inovação (Kirton, 1989).

\section{Participantes}

Utilizou-se uma amostragem por conveniência constituída a partir dos estudantes de turmas pré existentes. Participaram no estudo 131 alunos do $3^{\circ}$ ciclo do Ensino Básico, distribuídos pelos $7^{\circ}, 8^{\circ}$ e $9^{\circ}$ anos de escolaridade. O grupo experimental era constituído por 67 estudantes e o de controlo por 64. A variável género foi controlada em cada grupo (experimental: 36 raparigas e 31 rapazes; controlo: 36 raparigas e 28 rapazes). Os alunos tinham idades entre os 12 e os 15 anos $(\mathrm{M}=13,08$; $\mathrm{DP}=0,888)$, sendo oriundos de duas escolas privadas, nomeadamente do Porto e de Lisboa (Porto: 39 alunos no grupo experimental e 40 no de controlo; Lisboa: 28 alunos no grupo experimental e 24 no de controlo).

\section{Instrumento}

Foi aplicado o TTCT -Testes de Pensamento Criativo de Torrance, Versão Figurativa (Forma A), adaptada a Portugal para alunos entre o $5^{\circ}$ e o $9^{\circ}$ ano de escolaridade (Azevedo, 2007). É constituído por três atividades. Na primeira, há uma figura-estímulo para, a partir dela, se desenvolver um desenho; na segunda, são apresentadas 10 figuras para completar; na terceira, 30 pares de linhas paralelas são estímulo para o desenvolvimento de desenhos. Para cada desenho é ainda pedido um título. Cada tarefa dura 10 minutos. As competências criativas a avaliar são a Fluência (número de respostas relevantes), Originalidade (raridade das respostas), Elaboração (exposição de detalhes), Abstração dos Títulos (implicando síntese e organização de informação e 
sendo esta a única medida verbal), Resistência ao Fechamento (abertura no processamento da informação a partir de um estímulo) e Forças Criativas (parâmetro agregador de indicadores emocionais característicos das pessoas criativas: Expressão Emocional, Contando uma História, Movimento, Expressividade nos Títulos, Síntese de Figuras Incompletas, Combinação de Linhas ou Círculos, Perspetiva Invulgar, Visualização Interna, Extensão dos Limites, Humor, Riqueza de Imaginário, Coloração do Imaginário e Fantasia). É ainda calculável uma nota genérica para a criatividade do indivíduo avaliado: o Índice Criativo.

A versão usada demonstra boas qualidades psicométricas. $\mathrm{O}$ acordo inter-corretores indicou valores entre .66 e .90, valores próximos dos encontrados pelo autor do teste (Azevedo, 2007). A consistência interna emergiu como aceitável (Alfa de Cronbach de 0,70), de acordo com investigações sobre avaliação da criatividade e especificamente neste teste (Kim, Cramond e Bandalos, 2006). Obteve-se uma estrutura interna de dois fatores explicativos de $68,97 \%$ da variância dos resultados que, na opinião de Kim, Cramond e Bandalos (2006) podem ser contextualizados na teoria dos estilos de criatividade e de resolução de problemas Adaptação/Inovação (Kirton, 1989).

\section{Procedimentos}

Para a avaliação do programa de intervenção, recorreu-se a um grupo de controlo e a um grupo experimental, em dois momentos de recolha de informação (pré e pós teste). Os grupos foram avaliados no mesmo período de tempo e com o mesmo espaçamento entre os dois momentos. A avaliação foi efetuada em sala de aula e por um psicólogo. Anteriormente à pré-avaliação, foi solicitada permissão das escolas envolvidas e dos encarregados de educação, tendo-se comunicado sucintamente a todos os envolvidos o objetivo da administração das provas (estudo acerca da criatividade). A todos os participantes foi ainda garantida confidencialidade dos resultados e pedido o consentimento informado para a participação voluntária.

A aplicação do programa foi realizada na modalidade Resolução Criativa de Problemas Globais Futuristas e durou sete meses com a frequência média de 45'por semana, em contexto extracurricular de clube escolar. A aplicação foi realizada pela primeira autora deste artigo e apoiada por dois professores (um em cada escola) em momentos extrasessão (por exemplo, para tirar dúvidas ou monitorizar tarefas propostas para além das sessões), sendo todas as sessões planificadas em conjunto.

\section{Resultados}

Neste estudo procedeu-se a análises comparativas dos resultados nas competências criativas avaliadas, obtidos pelos grupos de controlo e experimental em dois momentos (antes e após a intervenção no grupo experimental), especificamente considerando: (1) percentis nacionais nas diversas competências criativas (para as Forças Criativas foram considerados valores brutos); (2) percentis nacionais para o Índice Criativo; (3) as dimensões Adaptação e Inovação. Tais resultados serão apresentados em diferentes secções.

\subsection{Competências criativas}

Foram comparados os resultados, atendendo aos percentis nacionais para cada uma das competências nos grupos de controlo e experimental e nos dois momentos. Para o 
parâmetro Forças Criativas, calculado a partir de vários indicadores, foram comparados valores brutos. Procedeu-se à análise de variância para medidas repetidas, cujos resultados se apresentam na tabela 1 .

Tabela 1. Comparação dos resultados para o $1^{\circ}$ e $2^{\circ}$ momentos de avaliação entre o grupo de controlo e o experimental

\begin{tabular}{|c|c|c|c|c|c|}
\hline COMPETÊNCIAS & GRUPO & $\begin{array}{c}1^{\circ} \text { Momento } \\
\text { M (DP) }\end{array}$ & $\begin{array}{c}2^{\circ} \text { MOMENTo } \\
\text { M (DP) }\end{array}$ & $\mathbf{F}$ & $\mathbf{P}$ \\
\hline \multirow{2}{*}{ Fluência } & Controlo & $60,83(26,729)$ & $62,00(27,104)$ & \multirow{2}{*}{85,237} & \multirow{2}{*}{0,000} \\
\hline & Experimental & $41,21(24,861)$ & $73,49(16,206)$ & & \\
\hline \multirow{2}{*}{ Originalidade } & Controlo & $25,52(19,842)$ & $23,50(20,581)$ & \multirow{2}{*}{40,139} & \multirow{2}{*}{0,000} \\
\hline & Experimental & $18,66(20,514)$ & $36,85(24,397)$ & & \\
\hline \multirow{2}{*}{ Abstração (nos títulos) } & Controlo & $46,1632,222)$ & $29,19(28,838)$ & \multirow{2}{*}{68,258} & \multirow{2}{*}{0,000} \\
\hline & Experimental & $50,05(30,400)$ & $68,09(26,657)$ & & \\
\hline \multirow{2}{*}{ Elaboração } & Controlo & $85,75(23,930)$ & $85,33(23,613)$ & \multirow{2}{*}{5,465} & \multirow{2}{*}{0,021} \\
\hline & Experimental & $92,03(16,984)$ & $97,21(6,986)$ & & \\
\hline \multirow{2}{*}{ Resistência fechamento } & Controlo & $53,57(30,666)$ & $47,78(28,360)$ & \multirow{2}{*}{25,123} & \multirow{2}{*}{0,000} \\
\hline & Experimental & $50,55(29,765)$ & $57,97(29,417)$ & & \\
\hline \multirow{2}{*}{$\begin{array}{l}\text { Forças criativas } \\
\text { (valores brutos) }\end{array}$} & Controlo & $12,17(4,997)$ & $9,80(4,332)$ & \multirow{2}{*}{33,532} & \multirow{2}{*}{0,000} \\
\hline & Experimental & $12,99(3,369)$ & $13,91(3,004)$ & & \\
\hline
\end{tabular}

Fonte: Elaboração própria.

Verificou-se que existem diferenças estatisticamente significativas entre os dois momentos quando comparados os valores dos percentis nacionais para os grupos de controlo e experimental (e os valores brutos para as Forças Criativas), face a todas as competências, ou seja, para todas elas houve um efeito de interação entre o momento e o grupo. Quando comparadas as médias de ambos os grupos em cada momento, constatase que para Fluência, Originalidade e Resistência ao Fechamento, o grupo de controlo apresenta média mais elevada do que o experimental no $1^{\circ}$ momento, enquanto nas restantes competências se verifica o inverso. Contudo, no $2^{\circ}$ momento as médias são sempre mais elevadas no grupo experimental. Comparadas as médias para cada grupo entre o $1^{\circ}$ e o $2^{\circ}$ momentos, verifica-se que a média do grupo de controlo apresenta valores mais baixos no $2^{\circ}$ momento com exceção na Fluência, a qual aumenta no $2^{\circ}$ momento. Porém, o grupo experimental apresenta médias mais elevadas em todas as competências no $2^{\mathrm{o}}$ momento.

Dos resultados da ANOVA para medidas repetidas, constata-se que existe um efeito principal significativo do momento nas competências de Fluência $(\mathrm{F}(1,129)=98,563$, $\mathrm{p}$ $=0,000)$, Originalidade $(\mathrm{F}(1,129)=25,723, \mathrm{p}=0,000)$, Elaboração $(\mathrm{F}(1,129)=3,942, \mathrm{p}$ $=0,049)$ e Forças Criativas $(\mathrm{F}(1,129)=6,469, \mathrm{p}=0,012)$, mas não na Abstração $(\mathrm{F}(1,129)=0,065, \mathrm{p}=0,800)$ nem na Resistência ao Fechamento $(\mathrm{F}(1,129)=0,380, \mathrm{p}=$ $0,539)$.

Também se constatou que existe um efeito principal significativo de grupo nas competências de Abstração $(\mathrm{F}(1,129)=20,017, \mathrm{p}=0,000)$, Elaboração $(\mathrm{F}(1,129)=$ $8,560, \mathrm{p}=0,004)$ e Forças Criativas $(\mathrm{F}(1,129)=14,940, \mathrm{p}=0,000)$, mas não na Fluência $(\mathrm{F}(1,129)=1,112, \mathrm{p}=0,294)$, Originalidade $(\mathrm{F}(1,129)=0,917, \mathrm{p}=0,340)$ e Resistência ao Fechamento $(\mathrm{F}(1,129)=0,511, \mathrm{p}=0,476)$. 
Existe ainda um efeito de interação significativo entre momento de intervenção e grupo (cf. tabela 1) em todas as competências estudadas. Estes resultados podem ser mais detalhadamente observados nas figuras 1 a 6 .

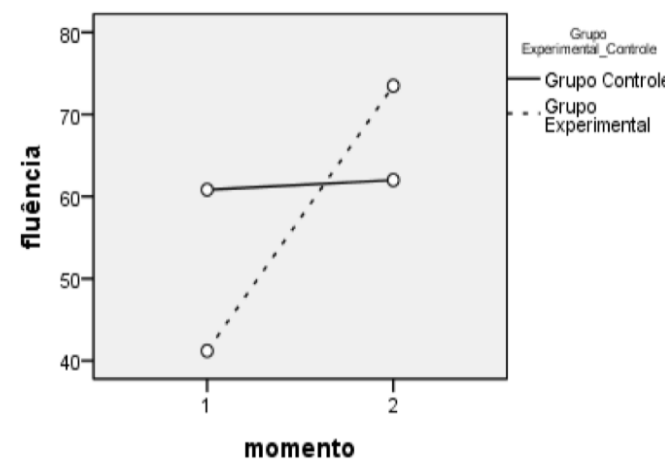

Figura 1. Dados da fluência em ambos os grupos e momentos

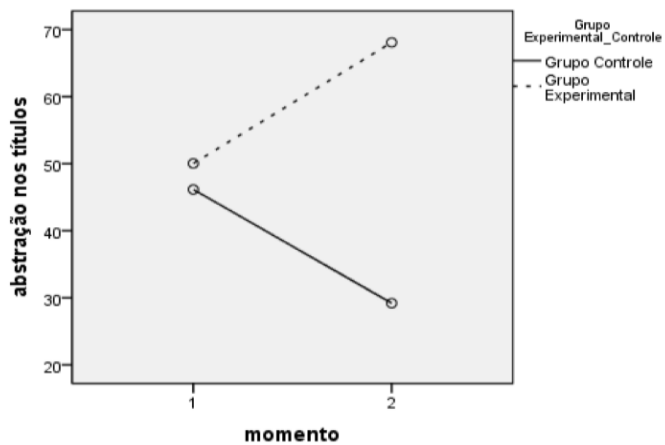

Figura 3. Dados da abstração nos títulos em ambos os grupos e momentos

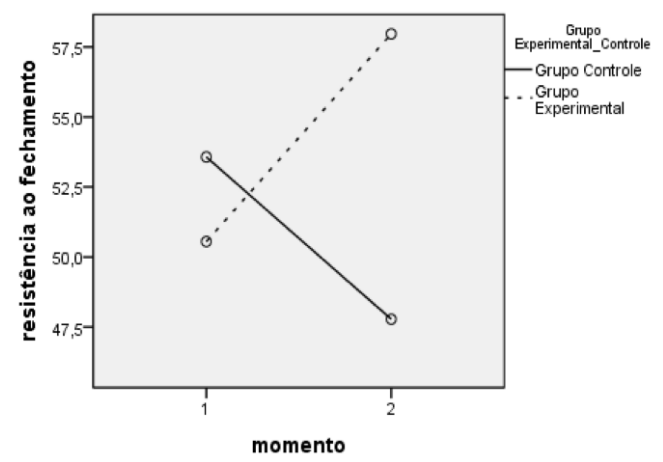

Figura 5. Dados da resistência ao fechamento em ambos os grupos e momentos

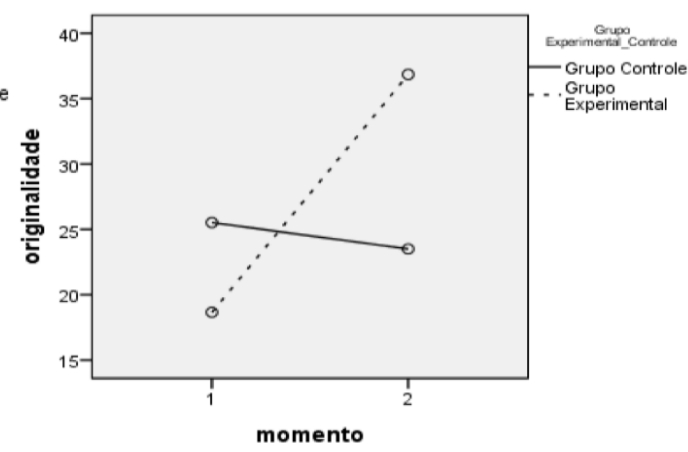

Figura 2. Dados da originalidade em ambos os grupos e momentos

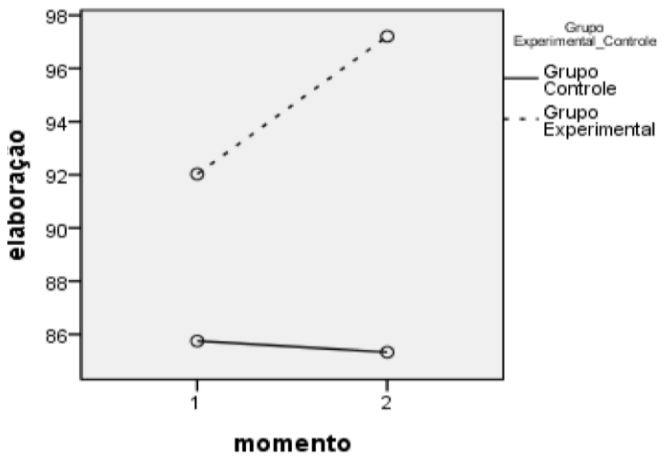

Figura 4. Dados da elaboração em ambos os grupos e momentos

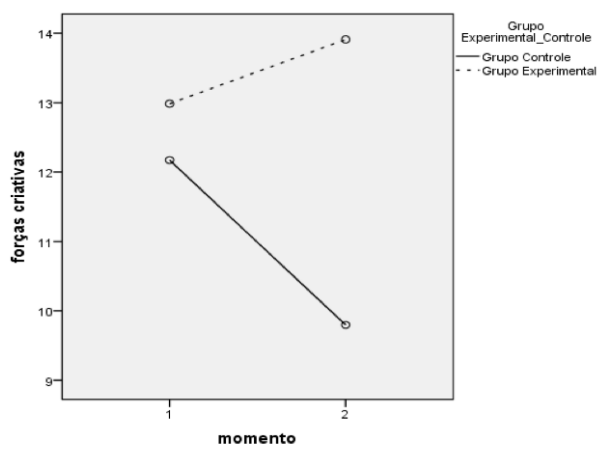

Figura 6. Dados das forças criativas em ambos os grupos e momentos

Fonte: Elaboração própria. 


\subsection{Classificação genérica de criatividade: índice criativo}

Tendo em conta os percentis nacionais, foram analisados os resultados do Índice Criativo, tomando os grupos de controlo e experimental nos dois momentos. Procedeuse à análise de variância para medidas repetidas, cujos resultados se apresentam na tabela 2.

Tabela 2. Comparação dos resultados no Índice criativo para o $1^{\circ}$ e $2^{\circ}$ momentos de avaliação entre o grupo de controlo e o experimental

\begin{tabular}{lcccc}
\hline \multicolumn{1}{c}{ GruPo } & $\mathbf{1}^{\circ}$ MOMENTO & $\mathbf{2}^{\circ}$ MOMENTO & \multirow{2}{*}{ F } & P \\
& M (DP) & M (DP) & & \\
\hline Controlo & $60,08(30,160)$ & $50,50(26,261)$ & \multirow{2}{*}{0,000} \\
Experimental & $59,43(27,283)$ & $82,70(16,504)$ & 96,793 & 0, \\
\hline
\end{tabular}

Fonte: Elaboração própria.

Verificou-se que existem diferenças estatisticamente significativas através de uma ANOVA medidas repetidas para o Índice Criativo (momento $\mathrm{x}$ grupo). O grupo de controlo mostra uma média superior ao grupo experimental no $1^{\circ}$ momento, mas no $2^{\circ}$ momento já é o grupo experimental a mostrar realização superior. Considerando os momentos de intervenção, o grupo experimental aumentou a realização, ao contrário do grupo de controlo.

Constata-se a existência de um efeito principal significativo do momento de intervenção $(\mathrm{F}(1,129)=16,815, \mathrm{p}=0,000)$, bem como um efeito principal significativo do grupo $(\mathrm{F}(1,129)=14,567, \mathrm{p}=0,000)$ no Índice Criativo. Verificou-se ainda um efeito de interação significativo grupo x momento nos resultados do Índice Criativo (cf. tabela 2). Estes resultados podem ser mais detalhadamente observados na figura 7 .

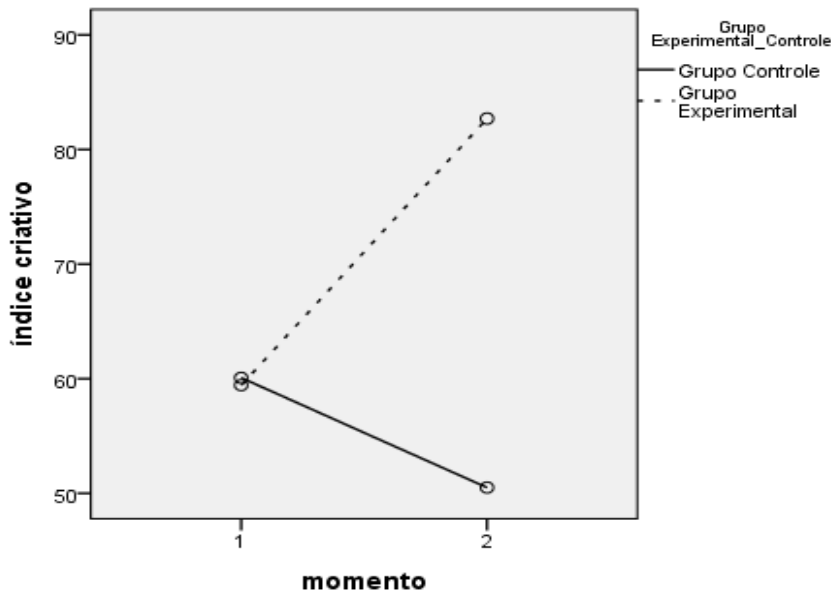

Figura 7. Dados do Índice Criativo em ambos os grupos e momentos Fonte: Elaboração própria.

\subsection{Estilos de resolução de problemas: adaptação e inovação}

Foram analisados ainda os resultados nas dimensões Adaptação (Abstração nos Títulos, Forças Criativas e Elaboração) e Inovação (Originalidade, Resistência ao Fechamento e Fluência), tomando os grupos de controlo e experimental, assim como os dois 
momentos. Os resultados da comparação através de uma análise de variância para medidas repetidas estão apresentados na tabela 3.

Tabela 3. Comparação dos resultados em Adaptação e Inovação para o $1^{\circ}$ e $2^{\circ}$ momentos de avaliação entre o grupo de controlo e o experimental

\begin{tabular}{|c|c|c|c|c|c|}
\hline DiMENSÃo & GRUPO & $\begin{array}{c}1^{\circ} \text { Momento } \\
\text { M (DP) }\end{array}$ & $\begin{array}{c}2^{\circ} \text { MOMENTO } \\
\text { M (DP) }\end{array}$ & $\begin{array}{c}\mathrm{F} \\
(1,129)\end{array}$ & $\mathbf{P}$ \\
\hline \multirow{2}{*}{ Adaptação } & Controlo & $31,27(9,620)$ & $28,06(7,850)$ & \multirow{2}{*}{86,341} & \multirow{2}{*}{0,000} \\
\hline & Experimental & $34,06(9,052)$ & $39,18(8,719)$ & & \\
\hline \multirow{2}{*}{ Inovação } & Controlo & $45,61(12,742)$ & $45,31(12,724)$ & \multirow{2}{*}{71,487} & \multirow{2}{*}{0,000} \\
\hline & Experimental & $39,42(11,827)$ & $51,97(9,117)$ & & \\
\hline
\end{tabular}

Fonte: Elaboração própria.

As diferenças encontradas são estatisticamente significativas para ambas as dimensões, comparados os grupos de controlo e experimental nos dois momentos estudados através de uma ANOVA para medidas repetidas. Na Adaptação, o grupo de controlo apresenta resultados mais baixos do que o experimental no $1^{\circ}$ momento e inversamente no $2^{\circ}$ momento. No $2^{\circ}$ momento, os resultados do grupo de controlo baixam, enquanto os do grupo experimental aumentam, face ao $1^{\circ}$ momento. Relativamente à Inovação, é o grupo de controlo que apresenta resultados mais elevados no $1^{\circ}$ momento, os quais baixam ligeiramente no $2^{\circ}$ momento. Os valores do grupo experimental, sendo mais baixos no $1^{\circ}$ momento, sobem no $2^{\circ}$ momento.

Dos resultados da ANOVA para medidas repetidas contata-se que existe um efeito principal significativo do momento na dimensão Adaptação $(\mathrm{F}(1,129)=4,577, \mathrm{p}=0,034)$ bem como na dimensão Inovação $(F(1,129)=65,033, p=0,000)$.

Existe ainda um efeito de interação significativo entre momento de intervenção e grupo (cf. Tabela 3) em ambas as dimensões. Estes resultados podem ser mais detalhadamente observados nas figuras 8 e 9 .

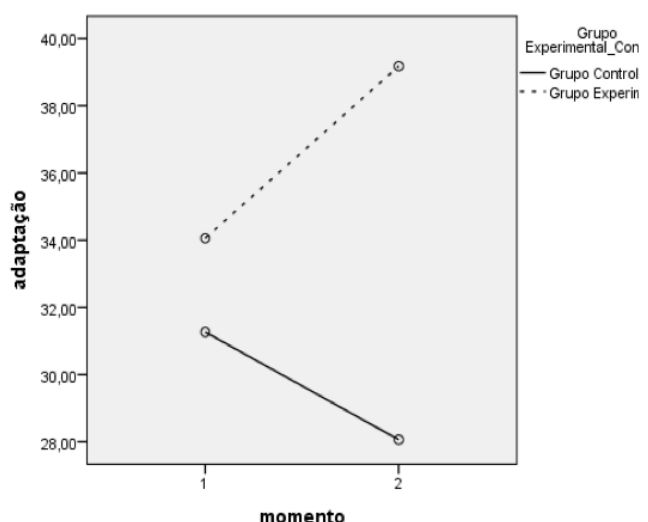

Figura 8. Dados da adaptação em ambos os grupos e momentos

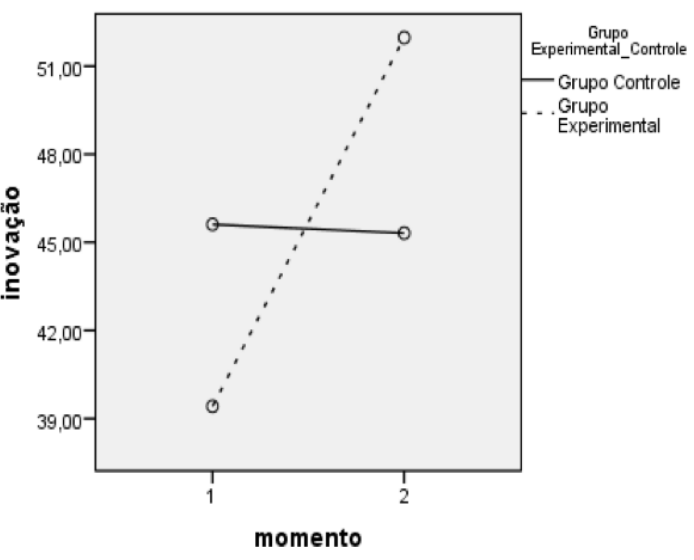

Figura 9. Dados da inovação em ambos os grupos e momentos

Fonte: Elaboração própria. 


\section{Discussão e conclusões}

$\mathrm{Na}$ atualidade surge como particularmente relevante a promoção de competências criativas no domínio educacional (Trilling e Fadel, 2012). Na diversidade de propostas de intervenção nesse sentido e nesse domínio, um programa que se tem destacado pela multiplicidade internacional da sua aplicação é o Future Problem Solving Program International - FPSPI (Torrance, Torrance, Williams e Horng, 1978).

Nesta investigação, avaliou-se o efeitos do FPSP em adolescentes, tomando várias competências criativas, de foro cognitivo e emocional, avaliadas pelos Testes Figurativos de Torrance adaptados a Portugal (Azevedo, 2007). Tendo em conta os momentos de pré e de pós intervenção, assim como os grupos experimental e de controlo, os resultados indicam existirem interações estatisticamente significativas entre grupo e momento para todas as dimensões avaliadas. As hipóteses inicialmente colocadas foram verificadas, mostrando que o programa de intervenção teve um efeito positivo nos seus participantes.

Nos resultados, o facto de o grupo experimental partir para a intervenção com um valor mais elevado em Elaboração e em Abstração nos Títulos, face ao de controle, não põe em causa o efeito positivo do programa neste estudo, já que após a intervenção todas as competências demonstraram aumento significativo no grupo experimental, mesmo na Fluência, competência na qual o grupo de controle detinha valor mais elevado no préteste. Também a classificação global de criatividade fornecida por esta avaliação ilustrou significativamente desenvolvimento no grupo experimental.

A noção de que criatividade é passível de treino (Runco, 2014) e a de que intervenções sistemáticas podem contribuir para tal promoção (Ma, 2006) são afirmadas na literatura. Os resultados desta investigação vêm enquadrar-se então neste cenário, mostrando desenvolvimento de competências cognitivas quer de âmbito cognitivo quer emocional. Focando o programa avaliado, efeitos positivos têm já sido encontrados nestes dois domínios (eg. Cramond e Fairweather, 2013; Treffinger, Selbye e Crumel, 2012).

Tomadas especificamente as competências avaliadas pelo TTCT neste estudo, é de referir que uma investigação conduzida pela STS -Scholastic Testing Service nos Estados Unidos (Kaufman, 2012; Woythal, 2014), a qual continua em curso, estas mesmas competências também emergiram como favorecidas pelo FPSP. Alunos dos $5^{\circ}$ e $6^{\circ}$ anos de escolaridade obtiveram melhor Indice Criativo no TTCT face a um grupo de controlo (Kaufman, 2012). A mesma amostra, no 7 e $8^{\circ}$ anos de escolaridade, voltou a demonstrar diferenças significativas face ao grupo de controlo no que respeita especificamente a Fluência, Originalidade, Abstração nos Títulos e Resistência ao Fechamento (Woythal, 2014). Em Portugal, Alves (2013) encontrou melhorias significativas na Fluência e na Originalidade quando aplicou o FPSP a adolescentes de cursos profissionais.

Este estudo vem então reforçar os dados anteriores no sentido de que o FPSP se apresenta como uma ferramenta que educa para a criatividade, tendo-se nele também observado especificamente a promoção de competências criativas traduzidas por indicadores emocionais (agrupados nas Forças Criativas). Parece compreensível este dado se for recordado que a metodologia de Resolução Criativa de Problemas, presente no FPSI, intervém na componente afetiva da aprendizagem, incluindo a aplicação de ferramentas como o jogo e a simulação, metáforas e analogias, sessões de imagética ou a 
redução de bloqueios emocionais, ferramentas que obrigam mentores e participantes jovens a explorar dimensões pessoais e interpessoais.

Por seu lado, os resultados positivos obtidos, tal como os de investigações já referidas, demonstram o desenvolvimento de criatividade em participantes adolescentes. Recordando a trajetória desenvolvimental deste constructo (Guinard e Lubart, 2006; Torrance, 1976), pode-se então pensar o FPSP como uma possibilidade interventiva no sentido de contrariar a tendência de decréscimo no início da adolescência e de potenciar crescimento no decurso desta mesma faixa etária.

Outro resultado interessante diz respeito às dimensões de Adaptação/Inovação, as quais agrupam competências criativas avaliadas pelo TTCT e parecem corresponder aos estilos de criatividade e de resolução de problemas propostos por Kirton (1989), segundo Azevedo (2007) ou Kim, Cramond e Bandalos (2006). Note-se que Kirton sublinha que ambos os estilos podem traduzir decisões e processos de realização criativa; estes apenas se diferenciam pelo facto de os adaptadores enfatizarem mais a precisão, a eficiência, o aproveitamento de conhecimento nesse processo, e os inovadores serem mais tendencialmente inconformados, questionadores de regras, procurando soluções diferentes para os problemas. O programa de intervenção, neste estudo, parece então sublinhar nos sujeitos os seus estilos próprios de serem criativos, possibilitando talvez um maior autoconhecimento sobre o processo individual de resolução de problemas, já que após a intervenção o grupo experimental exibe valores mais elevados em ambos os estilos face ao grupo de controlo.

Estudos com amostras maiores ou detalhando resultados em função de variáveis dos participantes ou da aplicação do programa enriquecerão certamente os resultados obtidos. Analisar as competências aqui avaliadas por anos de escolaridade poderia dar informações pertinentes sobre um eventual efeito diferencial do programa face aos períodos inicial e final da adolescência e assim ilustrar como pode rentabilizar mais a trajetória desenvolvimental da criatividade nesta idade. Também resultados em função do género podiam contribuir para o tópico ainda não pacífico da relação entre criatividade e essa variável (Kemmelmeier e Walton, 2012). A aplicação do FPSI a populações com especificidades como sobredotados ou alunos com dificuldades cognitivas e/ou emocionais, ou mesmo a sua aplicação comparada em modalidades diferentes ou em contextos de aplicação curricular/extracurricular, poderiam também rentabilizar o conhecimento que em diferentes países se deseja face à utilização deste programa. Espera-se, porém, que a presente investigação ajude a consolidar o sucesso deste programa, em geral e em Portugal, e, assim, a estimular mais desenvolvimentos sobre o seu estudo e uma sua aplicação mais frequente.

\section{Referências}

Alencar, E. M. L. S. (2015). Promoção da criatividade em distintos contextos: Entraves e desafios. En M. F. Morais, L. Miranda y S. Wechsler (Orgs.), Criatividade: Aplicações práticas em contextos internacionais (pp. 45-76). São Paulo: Vetor Editora.

Alves, M. M. (2013). Resolução criativa de problemas de âmbito comunitário em adolescentes do ensino profissional (Dissertação de mestrado). Universidade do Minho, Braga.

Alvino, J. (1993). Teaching our children to solve "fuzzy" problems. PTA Today, 18, 13-14. 
Azevedo, I. (2007). Criatividade e percurso escolar: Um estudo com jovens do ensino básico (Dissertação de doutoramento). Universidade do Minho, Braga.

Cramond, B. L. (2009). Future problem solving in gifted education. En L. Shavinna (Ed.), Handbook on giftedness (pp. 1143-1156). Nueva York, NY: Springer.

Cramond, B. L. e Fairweather, E. C. (2013). Future problem solving as education for innovation. En L. V. Shavinina (Ed.), The Routledge international handbook of innovation education (pp. 215-226). Londres: Routledge.

Guilford, J. P. (1950). Creativity. American Psychologist, 5, 444-454.

Guinard, J. H. e Lubart, T. (2006). Is it reasonable to be creative? En J. C. Kaufman y J. Baer (Eds.), Creativity and reason in cognitive development (pp. 269-281). Cambridge: Cambridge University Press.

Isaksen, S. G., Dorval, K. B. e Treffinger, D. J. (2011). Creative approaches to problem solving: a framework for change. Thousand Oaks, CA: Sage.

Kaufman, J. (2012). Future problem solving program international: brief summary of year one for longitudinal study. Recuperado de http://www.fpsp.org/impact.html

Kemmelmeier M. e Walton A. P. (2012). Creativity in its social context: The interplay of organizational norms, situational threat, and gender. Creativity Research Journal, 24(3), 208-2 19. doi:10.1080/10400419.2012.677345

Kim, K., Cramond, B. e Bandalos, D. (2006). The latent structure and measurement invariance of scores on the Torrance tests of creative thinking-figural. Educational and Psychological Measurement, 66(3), 459-477. doi:10.1177/0013164405282456

Kirton, M. J. (1989). Adaptors and innovators: Styles of creativity and problem solving. Londres: Routledge.

Ma, H. (2006). A synthetic analysis of the effectiveness of single components and packages in creativity training programs. Creativity Research Journal, 18, 435-446. doi:10.1207/s15326934crj1804_3

Masini, E. (2011). How to teach futures studies: Some experiences. Journal of Futures Studies, 15(4), 111-120.

Miller, A. L. e Mumford, A. D. (2014). Creative cognitive processes in higher education. The Journal of Creative Behaviour, 48, 1-17. doi:10.1002/jocb.77

Morais, M. F., Jesus, S., Azevedo, I., Araújo, A. M. e Viseu, J. (2015). Intervention program on adolescent's creativity representations and academic motivation. Paidéia, 25(6), 289-297. doi:10.1590/1982-43272562201502

Runco, M. A. (2014). Creativity: Theories and themes. Research, development and practice. San Diego, CA: Academic Press.

Runco, M. e Jager, G. (2012). The standard definition of creativity. Creativity Research Journal, 24(1), 92-96. doi:10.1080/10400419.2012.650092

Scott, G., Leritz, L. E. e Mumford, M. D. (2004). The effectiveness of creativity training: A quantitative review. Creativity Research Journal, 16, 361-388. doi:10.1207/s15326934crj1604_1

Torrance, E. P. (1976). Criatividade: Medidas, testes e avaliações. São Paulo: Ibrasa.

Torrance, E. P., Torrance, L. P., Williams, S. J. e Horng, R. Y. (1978). Handbook for training future problem solving. Athens, GA: University of Georgia. 
Treffinger, D., Selby, E. e Crumel, J. (2012). Evaluation of the future problem solving program international (FPSPI). IJCPS-International Journal of Creativity and Problem Solving, 22(2), $45-61$.

Trilling, B. e Fadel, C. (2012). 21st century skills: Learning for life in our times. Nueva York, NY: Jossey Bass.

Volk, V. (2008). A global village is a small world. Roeper Review, 30, 39-44. doi:10.1080/02783190701836445

Woythal, D. (2014). So... how well are we doing in meeting these goals? Recuperado de http://www.fpspi.org/newsletters.html

\section{Breve CV de las autoras}

\section{Ivete Azevedo}

Pós Doutorada em Educação pela Universidade do Minho; investigadora no âmbito da avaliação e desenvolvimento da criatividade no contexto educativo, tendo publicações a nível nacional e internacional; Presidente da Direção e sócia fundadora do Torrance Center Portugal; Diretora para Portugal do FPSPI (Future Problem Solving Program International). ORCID ID: 0000-0002-5327-4211. Email: iveteazevedo@tcportugal.org

\section{Maria de Fátima Morais}

Licenciada em Psicologia pela Universidade do Porto e doutorada em Psicologia da Educação pela Universidade do Minho. Nesta última Universidade, é Professora Auxiliar do Instituto de Educação, lecionando disciplinas de graduação e de pósgraduação. É membro integrado do Centro de Investigação em Educação (CIEd) na Universidade do Minho. É membro da Associação Nacional para o Estudo Intervenção na Sobredotação (ANEIS) e da Rede Internacional de Investigação, Intervenção e Avaliação em Altas Capacidades Intelectuais (REINEVA). Faz parte da Conselho Consultivo Científico-Pedagógico do Torrance Center Portugal. É membro do Conselho Científico e Editorial de algumas revistas. O seu domínio de investigação é o da criatividade em contexto educativo, sendo autora e co-autora de várias escalas de avaliação, tendo publicações a nível nacional e internacional e orientado teses de mestrado de doutoramento e de pos-doutoramento sobre essa temática. ORCID ID: 0000-000 1-8180-6687. Email: fatima.morais@mail.telepac.pt

\section{Fernanda Martins}

Foi professora de Psicologia no âmbito da Formação de Professores e responsável por essa área científica na Faculdade de Letras da Universidade do Porto. Possui mestrado e doutoramento em Psicologia. Desde 2005 leciona Metodologia de Investigação a alunos de Licenciatura, Mestrado e Doutoramento e Psicologia Cognitiva a alunos de Mestrado na mesma Faculdade na área de Ciência da Informação. Tem participado em projetos de investigação e tem publicado e apresentado os resultados da sua investigação em encontros científicos nacionais e internacionais. É membro da Ordem dos Psicólogos Portugueses, da American Psychological Association e da American Psychological Society. As principais áreas de interesse em investigação são a Psicologia Cognitiva, particularmente as emoções e sua interação com a cognição. ORCID ID: 0000-0003-1357-1665. Email: mmartins@letras.up.pt 